\title{
Cholinergic Stimulation Enhances Neural Activity Associated with Encoding but Reduces Neural Activity Associated with Retrieval in Humans
}

\author{
Juraj Kukolja, ${ }^{1,2}$ Christiane M. Thiel, ${ }^{2,3}$ and Gereon R. Fink ${ }^{1,2}$ \\ ${ }^{1}$ Department of Neurology, University Hospital, Cologne University, 50924 Cologne, Germany, ${ }^{2}$ Cognitive Neurology Section, Institute of Neuroscience and \\ Medicine (INM-3), Research Centre Juelich, 52425 Juelich, Germany, and ${ }^{3}$ Institute of Biology and Environmental Sciences, Carl von Ossietzky University \\ Oldenburg, 26111 Oldenburg, Germany
}

\begin{abstract}
The cerebral cholinergic system is centrally involved in memory formation. Studies in rodents suggest that cholinergic stimulation may facilitate encoding of new information but may interfere with retrieval. We investigated the effect of cholinergic stimulation on encoding and retrieval of episodic memory in humans. We also tested whether the putative benefit of cholinergic stimulation on memory function depends on individual baseline performance. Since such effects were expected to be greatest in an older population resulting from an age-related degeneration of the cholinergic system, we recruited 22 healthy older subjects (51-68 years) for an event-related functional magnetic resonance imaging experiment. In two separate scanning sessions, subjects encoded and retrieved items and their spatial context under cholinergic stimulation or placebo with the acetylcholine-esterase inhibitor physostigmine or saline being administered intravenously in a double-blind cross-over design. Baseline performance was recorded at a separate occasion without scanning. Cholinergic stimulation enhanced neural activity for successful versus unsuccessful spatial context encoding in the right hippocampus but reduced activity for successful versus unsuccessful spatial context retrieval in the right amygdala. These data may bridge the gap between rodent and human studies by showing that also in man cholinergic stimulation enhances encoding but interferes with retrieval on a neural level. Furthermore, baseline performance negatively correlated with the effect of cholinergic stimulation. Thus, participants who were worse at baseline benefited more from cholinergic stimulation than those who had better baseline values, indicating that a cholinergic deficit contributes to the memory decline even in healthy older subjects.
\end{abstract}

\section{Introduction}

The cerebral cholinergic system is centrally involved in memory formation. Whether elevated acetylcholine (ACh) levels have beneficial or detrimental effects, however, critically depends on the phase of memory formation at which cholinergic stimulation occurs. During encoding, stimulation of nicotinic cholinergic receptors facilitates (Buccafusco et al., 2005; Levin et al., 2006), but muscarinic blockade (Hasselmo and McGaughy, 2004; Rasch et al., 2006) and combined muscarinic and nicotinic cholinergic blockade (Atri et al., 2004) impair human performance. In contrast, cholinergic stimulation with the cholinesterase inhibitor physostigmine, rather than enhancing, interferes with retrieval in animals (Rogers and Kesner, 2003), and muscarinic blockade has little effect on memory retrieval in humans (Thiel et al., 2002; Atri et al., 2004; Hasselmo and McGaughy, 2004). Such observations support the model proposed by Hasselmo et al.

Received Jan. 14, 2009; revised May 21, 2009; accepted May 22, 2009.

This work was supported by a grant of the Deutsche Forschungsgemeinschaft (DFG-KF0 112, TP8) to G.R.F. and C.M.T. We thank all our volunteers. We are grateful to our colleagues of the MR and Cognitive Neurology groups for valuable support. We thank Laura Amort and Birte Berger for neuropsychological testing.

The authors declare no conflict of interest.

Correspondence should be addressed to Dr. Juraj Kukolja, Department of Neurology, University Hospital, Cologne University, Kerpener Strasse 62, 50924 Cologne, Germany. E-mail: juraj.kukolja@uk-koeln.de. DOI:10.1523/JNEUROSCI.0203-09.2009

Copyright $\odot 2009$ Society for Neuroscience $\quad 0270-6474 / 09 / 298119-10 \$ 15.00 / 0$
(2006), suggesting that cholinergic stimulation enhances excitatory afferent input to memory relevant areas and at the same time disrupts neural interference by ongoing retrieval processes. Hence, cholinergic modulation may increase neural selectivity for incoming information and thereby enhance the efficiency of encoding at the cost of concurrent retrieval processes. Supporting this view, functional imaging data have shown that cholinergic stimulation during working memory tasks enhances activity in early visual processing regions, possibly facilitating encoding, but decreases activity in prefrontal cortex which mediates the maintenance of memorized information (Furey et al., 2000; Freo et al., 2005).

Central hubs for episodic memory encoding and retrieval, i.e., the hippocampal formation (Schacter and Wagner, 1999; Burgess et al., 2002) and the amygdala (Canli et al., 2000; McGaugh et al., 2002; McIntyre et al., 2003; Rutishauser et al., 2008), are major recipients of cholinergic fibers which originate in the subnuclei of the basal forebrain (Mesulam et al., 1983; Mesulam and Geula, 1988; Mufson et al., 2003; McGaugh, 2004). A premature degeneration of these cholinergic neurons is observed in Alzheimer's dementia (AD), which is characterized by a rapid decline in the ability to encode new information (Mesulam, 2004). Consequently, treatment of $\mathrm{AD}$ has focused on pharmacological enhancement of cholin- 
ergic function (Lanctôt et al., 2003; Trinh et al., 2003). Possibly, also the decline of memory functions observed during normal aging (Light, 1991; Spencer and Raz, 1995; NavehBenjamin, 2000; Kukolja et al., 2009) is based on a beginning cholinergic deficit (Bartus et al., 1982; Mesulam, 1996; Cohen et al., 2006; Schliebs and Arendt, 2006). Supporting this notion, nicotinic stimulation has been shown to enhance memory performance in healthy elderly adults in several tasks including verbal and object learning, delayed recall, and word retrieval (Howe and Price, 2001; Min et al., 2001). Since nicotine was applied chronically in these studies, the effects on encoding and retrieval were not distinguished. Furthermore, nicotine increased reaction times in a choice reaction time task, showing beneficial effects on attention in elderly subjects (White and Levin, 2004). However, a recently published study suggests that neural activity mediating selective attention is enhanced by cholinergic stimulation in AD patients but is disturbed in healthy older controls (Bentley et al., 2008). Possibly, the effect of cholinergic substitution depends on the integrity of the individual's cholinergic system.

The goal of the present study was to investigate the effects of cholinergic enhancement on activity in medial temporal lobe structures mediating encoding and retrieval of item and spatial contextual memory. According to the model proposed by Hasselmo and et al. (2006), we hypothesized that increasing the ACh level would enhance neural activity for encoding but leave unaltered or even impair activity-mediating retrieval. Using event-related functional magnetic resonance imaging (fMRI), we tested this hypothesis in healthy older subjects in which we expected a largely intact, albeit, compared with younger adults, functionally compromised cholinergic system.

\section{Materials and Methods}

Subjects. Twenty-two older subjects participated in the present study. Two subjects were subsequently excluded because of side effects (i.e., nausea and vomiting). One subject was excluded because of guessing in item recognition, and another subject was excluded because of a previously undetected but asymptomatic leukoencephalopathy.

Thus, 18 subjects ( 5 female and 13 male, aged 51-68 years), all without history of neurological or psychiatric disease were included in the present analysis. All were right handed, as assessed by the Edinburgh Handedness Inventory (Oldfield, 1971), with a mean laterality quotient of $+83.4 \pm 25.5$. All subjects had normal or corrected-to-normal vision. Informed written consent was given by all subjects before participation. Ethics approval was obtained from the local ethics committee. Before participation, subjects underwent a physical exam by an experienced physician (J.K.) to exclude any medical condition forbidding physostigmine administration or MRI scanning.

All subjects underwent neuropsychological assessment by use of the Consortium to Establish a Registry of AD (CERAD) test battery including the Mini-Mental State Examination [MMSE, German version (Kessler et al., 1990)], verbal fluency (telling as many animal names as possible within $1 \mathrm{~min}$ ), and measures of verbal learning and memory: word list memory test (VLMT), in which a list of 10 words was read aloud three times to the subjects who were asked to repeat the words after each presentation. The sum of all correctly repeated words comprised the immediate recall score. After $30 \mathrm{~min}$, subjects were asked to recall the words previously encoded (free recall), and were then presented with a word list containing the 10 previously presented words in addition to 10 new words (recognition/correct rejection) [normative data derived from Satzger et al. (2001)]. Furthermore, we tested for verbal association fluency [writing down as many words with the initial letters F, A, or S (Benton and Hamsher, 1976)], for depressive symptoms using the Beck Depression Inven-
Table 1. Group specifications and neuropsychological test results

\begin{tabular}{lcc}
\hline & Group characteristics & \\
\cline { 2 - 3 } & Mean (SD) & Normals (SD) \\
\hline Age & $58.2(5.0)$ & n.a. \\
Male & 13 & n.a. \\
Female & 5 & n.a. \\
Years of education & $15.5(3.2)$ & n.a. \\
MWT-B: IQ estimate & $124.8(10.6)$ & $100(15.0)$ \\
CERAD: verbal fluency & $25.0(5.2)$ & $22.0(4.8)$ \\
CERAD: MMSE & $29.5(1.0)$ & $29.0(1.0)$ \\
CERAD: VLMT immediate total & $23.3(3.2)$ & $22.4(3.4)$ \\
CERAD: VLMT free recall & $8.4(2.0)$ & $7.8(1.7)$ \\
CERAD: VLMT recognitions & $9.9(0.3)$ & $9.4(1.0)$ \\
FAS & $38.1(6.9)$ & $42.1(11.1)$ \\
HAWIE-R: digit span & $11.7(3.5)$ & $10.0(3.0)$ \\
BDI & $2.5(3.0)$ & $6.5(5.2)$ \\
MAC-0 & $24.1(4.8)$ & $<25$ \\
\hline
\end{tabular}

Specified are mean scores and age-corrected control data (SD) provided by the respective test manuals and studies reporting normative data (see Materials and Methods). FAS, Verbal association fluency (letters F, A, S); n.a., not applicable.

tory (BDI) (Beck et al., 1995), or subjective memory complaints using the Memory Assessment Clinics Questionnaire (MAC-Q) (Crook, III et al., 1992), for verbal intelligence quotient [MehrfachwahlWortschatz Test (MWT-B), in which subjects had to indicate real words within lists of pseudo-words (Lehrl, 1995)] and for working memory [digit span, Revised Hamburg Wechsler Intelligence Exam (HAWIE-R) (Tewes, 1991)] (Table 1). The digit span test consisted of seven runs during which subjects had to repeat two sequences of numbers forward and seven runs during which number sequences had to be repeated backward. The raw score (range 0-28) was then transformed to a normalized test score ranging from 1 to 19 , the results of which are reported in Table 1 . All subjects performed within normal ranges in each test (Table 1). In the MAC-Q, nine subjects had a score of 25 or more, indicating a subjective memory impairment.

Procedure. In two scanning sessions that were 1 week apart, subjects received either physostigmine by continuous intravenous infusion or the same amount of saline (as placebo) via an infusion system situated outside the scanner room (Perfusor fm, B. Braun Melsungen AG) in a double-blind cross-over design. In the drug condition, the physostigmine infusion $(1 \mathrm{mg} / \mathrm{h})$ was applied according to a protocol similarly used in previous experiments (Furey et al., 2000; Bentley et al., 2004): after a $10 \mathrm{~min}$ loading dose administered at $1.930 \mathrm{mg} / \mathrm{h}$ to quickly achieve the desired plasma levels, the infusion was continued at a rate of $0.816 \mathrm{mg} / \mathrm{h}$ to maintain stable drug levels for the remaining part of the experiment. Before the physostigmine infusion, glycopyrrolate $(0.2 \mathrm{mg})$ was intravenously injected to prevent peripheral cholinergic side effects. In the placebo condition, the same amounts of saline were injected. Before drug (physostigmine/placebo) administration and after scanning, electrocardiogram (ECG) recordings were performed on each subject to measure heart rate and to exclude ECG abnormalities. Furthermore, heart rate was monitored using pulse oximetry throughout scanning. Scanning started $30 \mathrm{~min}$ after infusion onset. After each scanning session, subjects were given a questionnaire assessing adverse reactions to physostigmine or glycopyrrolate (or placebo).

We used a spatial source memory task used previously by Cansino et al. (2002) and Kukolja et al. (2008, 2009). Briefly, the experiment consisted of an encoding and a retrieval session. During both encoding and retrieval, fMRI measurements took place. Stimuli to be encoded and retrieved were color photographs of natural and artificial objects that were presented on a LCD (liquid crystal display) screen behind the MRI scanner. The baseline display consisted of a green cross, which divided the screen into four quadrants. A small red cross was superimposed in the middle and served as fixation point. During the encoding session, 64 stimuli were selected for each subject from a pool of 96 stimuli. The stimuli appeared randomly for $2000 \mathrm{~ms}$ in one of the four quadrants of the screen and were followed by an inter- 
stimulus interval of $1450 \mathrm{~ms}$. The subjects were told to memorize each item and the position where it appeared. To ensure sufficient processing and recognition of the stimuli, subjects had to indicate with the index and middle finger of their right-hand whether the object was "natural," e.g., an animal or a vegetable, or whether it was "artificial," e.g., a tool or an instrument.

During the retrieval run, all 96 stimuli were presented randomly at the center of the screen. Thus, in addition to the 64 objects previously shown during the encoding session, 32 new objects were interspersed. Stimuli were presented for $1500 \mathrm{~ms}$, followed by a $2650 \mathrm{~ms}$ interstimulus interval. Responses were recorded with two button pads, one for each hand. The subjects' task was to decide whether each picture presented was novel or had been previously presented during the encoding run. If the item was classified as new, subjects pressed a button with their left index finger, and if the item was classified as previously seen, subjects used their right hand to indicate the position on the screen where the stimulus had appeared in the encoding session by pressing one of four respective keys (index to little finger; spatial context judgment). Subjects were instructed to make a guess regarding the position of the object if they were not sure about the position. To obtain a measure for baseline performance and to avoid training effects between the first and second scanning sessions, subjects performed the task in a training session on a separate occasion before the scanning sessions, which equalled the task during scanning in amount and duration. For the three sessions, three different sets of stimuli were used.

The durations of the encoding and retrieval sessions were 6.1 and $10.4 \mathrm{~min}$, respectively. There was a $\sim 4 \mathrm{~min}$ pause between the sessions during which subjects maintained their position in the scanner. In both sessions, $1 / 3$ of the stimuli were null events in which merely the baseline picture was shown. This effectively resulted in variable stimulus-onset asynchronies and allowed a comparison of the blood oxygen level dependent (BOLD) signal of the event types of interest with a no-stimulus baseline. Before the fMRI experiment, subjects performed one training session outside and one inside the MR scanner with short versions of the encoding and retrieval tasks.

Behavioral data analysis. Behavioral data were analyzed with respect to reaction times (RTs) and response accuracy. The analysis focused on items that were correctly recognized as previously seen in the retrieval session. These items were divided into two subtypes during encoding: (1) those which were assigned to the correct spatial position during the subsequent retrieval session [thus, correct spatial context encoding (CorSCE)], and (2) those which were assigned to a false spatial position during subsequent retrieval [false spatial context encoding (FalSCE)]. Accordingly, in the retrieval session, items were divided into two subtypes: (3) those with those with correct spatial context retrieval (CorSCR) and (4) items with false spatial context retrieval (FalSCR). In some of the analyses, the CorSCR and FalSCR were pooled to reflect item memory rather than spatial context memory (see also Kukolja et al., 2009). All data were analyzed with repeated measures ANOVAs and drug (placebo, physostigmine) as within subject factor. The order of physostigmine or placebo administration was included into the analyses as a covariate. Note that the same applies to the analysis of the imaging data.

To investigate whether the effect of cholinergic enhancement on behavioral measures depended on individual baseline performance, we correlated the performance during the baseline session with the effect of cholinergic enhancement in item and spatial context memory [i.e., correlation of $\Delta[$ CorSCR(physostigmine) - CorSCR(placebo)] with CorSCR (baseline), and correlation of $\Delta$ [all recognized items (physostigmine) - all recognized items (placebo)] with all recognized items (baseline), respectively.

Several diagnostic tests were applied to the data to verify the validity of a multiple regression analysis. A Kolmogorov-Smirnov goodness-of-fit test with Lilliefors significance correction (item recognition: statistic $=0.103, \mathrm{df}=16$, significance $=0.200$; CorSCR: statistic $=0.113, \mathrm{df}=16$, significance $=0.200)$ and a Shapiro-Wilk test (item recognition: statistic $=0.967, \mathrm{df}=16$, significance $=0.791$; CorSCR: statistic $=0.941, \mathrm{df}=16$, significance $=0.367)$ could not reject the null hypothesis that the standardized residuals of item recognition and CorSCR were normally distributed, fulfilling the requirements for the multiple regression analysis (Rawlings et al. 2001). None of the values for baseline item recognition or drug effect on item recognition, or CorSCR or drug effect on CorSCR, could be regarded as univariate outliers since all values fell into a range set by $1.5 \times$ the respective interquartile range below the first or above the third quartile. By calculation of the Mahalanobis distance (Mahalanobis, 1936), it was assured that none of the data points were to be regarded as multivariate outliers [Mahalanobis distance range for item recognition: $0.56-8.17$, critical value $\chi^{2}(0.001,3)=16.27$; Mahalanobis distance range for CorSCR: $0.56-5.11$, critical value $\left.\chi^{2}(0.001,3)=16.27\right]$. Furthermore, the calculation of Cook's distance revealed that none of the data points exerted an excessive influence on the regression analysis (Cook's distance range item recognition: $0.00-0.37$, critical value $F_{(0.5,3,13)}=0.83$; Cook's distance range for CorSCR: $0.00-0.77$, critical value $F_{(0.5,3,13)}=0.83$ ) (Rawlings et al., 2001).

Analogous to the repeated measures ANOVA, the order of drug administration was included as a covariate. Since the baseline session was independent of the placebo or physostigmine sessions, this approach was resistant against statistical artifacts such as a regression to the mean, which would have confounded a simple correlation of results during the physostigmine condition with those during placebo. One subject was excluded from the regression analysis because of an outlying baseline value ( $>3$ SDs below the group mean). A further subject was excluded because of computer failure to record responses during the baseline session. Thus, the regression analysis was performed in the remaining 16 subjects.

In an exploratory analysis, we additionally correlated the behavioral results (CorSCR accuracy and item recognition) during the placebo session with the neuropsychological measures taken on a separate occasion, to investigate whether the performance in the spatial contextual memory paradigm depended on neuropsychological test scores.

Using point-biserial correlation analyses, we tested whether the drug effect on item memory and spatial context retrieval was associated with the presence or absence of side effects, or whether the occurrence of side effects depended on baseline performance.

$f M R I$ hardware and procedures. Functional MR images were acquired using a TRIO 3-T whole-body scanner (Siemens) equipped with a standard head coil for radiofrequency transmission and signal reception. Sequence parameters were: $\mathrm{T} 2^{\star}$-weighted echoplanar images $(\mathrm{EPI})$ with BOLD contrast, echo time $=30 \mathrm{~ms}$, repetition time $=$ $2200 \mathrm{~ms}$, flip angle $=90^{\circ}$, slice thickness $3.0 \mathrm{~mm}$, interslice gap 0.3 $\mathrm{mm}$, field of view $=200 \mathrm{~mm}$, matrix size $64 \times 64$, in-plane resolution $=3.125 \mathrm{~mm} \times 3.125 \mathrm{~mm}$. 36 axial slices per volume covering the brain from vertex to cerebellum were acquired sequentially. In addition, a high-resolution T1 anatomical image was obtained for each subject using a three-dimensional magnetization-prepared, rapid acquisition gradient echo (MP-RAGE) sequence.

The fMRI time series consisted of 168 images for the encoding session and 285 images for the retrieval session, respectively. The first five images of the encoding session and the first seven images of the retrieval session were discarded from analysis to allow for the MR signal to reach a steady state.

Image processing. The image preprocessing was performed using Matlab7 (The MathWorks) and SPM5 (statistical parametric mapping software, Wellcome Department of Imaging Neuroscience, London, UK; http://www.fil.ion.ucl.ac.uk). The images of each time series were spatially realigned to the first image to correct for head movements between scans. The EPI images were then spatially normalized to the EPI template provided by SPM5 (resampled to $3 \times 3 \times 3 \mathrm{~mm}^{3}$ voxels) (Friston et al., 1995). Subsequently, EPI images were smoothed with a Gaussian kernel of $8 \mathrm{~mm}$ (full-width-halfmaximum) to compensate for normal variation in individual brain anatomy across subjects (Worsley and Friston, 1995).

Statistical analysis. Statistical analysis was performed using a mixed effects model. The two sessions (encoding, retrieval) were analyzed 
separately. For the encoding session, the data of each subject were analyzed by defining three event types at the first level of the mixed effects model, consisting of two effects of interest (CorSCE, FalSCE) and one effect of no interest (comprising items presented during encoding but which were classified as "new" during retrieval as well as missed responses in the encoding and retrieval sessions). For the retrieval session, three event types were defined at the first level: two effects of interest (CorSCR, FalSCR) and one effect of no interest (including items shown during encoding but which were falsely attributed to be "new" during retrieval, new items correctly or incorrectly responded to, and missed responses). The head movement parameters were included as additional regressors.

In the context of the GLM used by SPM5, we modeled the events as stick functions which were convolved with a canonical hemodynamic response function (HRF) and its first-order temporal derivative. The data were high-pass filtered to $1 / 128 \mathrm{~Hz}$ to remove subject-specific low frequency drifts in signal changes. The parameter estimates for the HRF and linear contrasts of these estimates comprised the data for the second level analysis.

At the second level, we performed an ANOVA for the encoding and retrieval session, respectively, with the within-subject factors spatial context judgment (correct, false) and drug (physostigmine, placebo). In analogy to the analysis of the behavioral data, order of drug administration was introduced as a nuisance covariate. To identify regions differentially active as a function of drug and/or spatial context judgment, $F$-tests were performed for the main effect of drug during encoding [physostigmine(CorSCE + FalSCE) $<>$ placebo(CorSCE + FalSCE)] and retrieval [physostigmine (CorSCR + FalSCR $)<>$ placebo(CorSCR + FalSCR)], the main effect of spatial context judgment during encoding [physostigmine(CorSCE) + placebo(CorSCE $)<>$ physostigmine(FalSCE) + placebo(FalSCE)] and retrieval [physostigmine $($ CorSCR $)+$ placebo(CorSCR) $<>$ physostigmine(FalSCR) + placebo(FalSCR)] and the interaction effects between drug and spatial context judgment during encoding [physostigmine(CorSCE $>$ FalSCE) $<>$ placebo(CorSCE $>$ FalSCE) $]$ and retrieval [physostigmine(CorSCR $>$ FalSCR) $<>$ placebo(CorSCR $>$ FalSCR)]. The latter comprised the primary focus of the analysis as they tested our hypotheses on cholinergic effects on neural activity mediating spatial context memory during encoding and retrieval. The tests resulted in a $F$-statistic for every voxel. The resulting set of voxel values for each contrast constituted a statistical parametric map of the $F$-statistic $\mathrm{SPM}_{\{F\}}$, which was subsequently transformed to the unit normal distribution $\mathrm{SPM}_{\{z\}}$. Post hoc $t$ tests for simple effects, if applicable, were performed within the clusters found significant in the ANOVA.

We restricted our analysis to the hippocampus and the amygdala. The hippocampal formation [including the cornu ammonis (CA1CA3), the dentate gyrus (including the fascia dentata and CA4), the subicular complex and the hippocampal-amygdaloid transition area] and the amygdalae (including the superficial, centromedial and laterobasal group of nuclei) bilaterally were defined as regions of interest (ROIs) based on cytoarchitectonic maximum probability maps (Amunts et al., 2005) using the SPM Anatomy toolbox [Version 1.4, http://www.fz-juelich.de/ime/spm_anatomy_toolbox (Eickhoff et al., 2005)]. These maps denote the most likely anatomical area at each voxel of the Montreal Neurological Institute (MNI; Montreal, QC, Canada) single subject template based on probabilistic cytoarchitectonic maps derived from a sample of 10 human postmortem brains, which were subsequently normalized to the MNI space (Amunts et al., 2005). The amygdaloid ROI used in the present study covered the centromedial, superficial, and laterobasal groups of amygdaloid nuclei (Amunts et al., 2005). Activations are reported at $p<0.05$, corrected for family-wise error (FWE), within the ROIs.

In an exploratory analysis, we tested whether the respective effect sizes (i.e., main and interaction effects) within the clusters found to be significantly active correlated with any neuropsychological test measure.

Localization of activations. The anatomical localization of significant activations and volume differences was assessed by reference to
Table 2. Behavioral data

\begin{tabular}{|c|c|c|c|c|}
\hline & Placebo & $\begin{array}{l}\text { Physostig- } \\
\text { mine }\end{array}$ & $F_{(1,16)}$ & $p$ \\
\hline Encoding: accuracy (\%) & $97.2(3.5)$ & $96.6(2.3)$ & 2.294 & 0.151 \\
\hline Retrieval: CorSCR (\%) & $48.6(18.0)$ & $40.4(15.3)$ & 3.627 & 0.075 \\
\hline Retrieval: CorSCR + FalSCR (\%) & $82.5(9.0)$ & $82.9(7.3)$ & 0.006 & 0.937 \\
\hline
\end{tabular}

Specified are mean percentage scores (SD). $p$ values reflect the results of univariate ANOVAs with the order of drug administration as a nuisance covariate. CorSCR represents percentage of items correctly recognized as previously seen and associated with a correct spatial context judgment. CorSCR + FalsSCR represent percentage of items correctly recognized as previously seen irrespective of the spatial context judgment (item memory).

the MNI standard stereotactic space that approximates the Talairach system (Talairach and Tournoux, 1988).

\section{Results}

\section{Behavioral results}

Encoding

Repeated measures ANOVAs on accuracy to classify objects as natural or artificial did not reveal any main effect of treatment (Table 2). Furthermore, repeated measures ANOVAs on RTs during encoding did not reveal any main effect of treatment, spatial context or any interaction between the two (all $p$ values $>0.05$ ).

\section{Retrieval}

We observed a nonsignificant trend for a reduction of the accuracy of spatial context retrieval under cholinergic stimulation. There was no effect of cholinergic stimulation on item recognition (values are listed in Table 2). Analysis of reaction times revealed a main effect of spatial context which was caused by faster RTs for CorSCR than for FalSCR items [CorSCR (mean \pm SD): $1459.75 \pm 240.69$ ms; FalSCR: $1588.68 \pm$ $\left.271.91 \mathrm{~ms}, F_{(1,15)}=12.70, p<0.005\right]$ but no main effect of drug and no interaction between spatial context and drug ( $p$ values $>0.05$ ).

A correlation analysis revealed that item recognition significantly correlated with immediate recall scores in the VLMT $(n=18, r=0.492, p<0.05)$ and with the BDI scores $(n=18$, $r=0.473, p<0.05)$. Item recognition scores were not correlated with any other neuropsychological test measures. Furthermore, the number of CorSCR responses was not correlated with any neuropsychological measure.

A multiple regression analysis was performed to test associations between the accuracy of item recognition at baseline [all recognized items (baseline)] and the effect of cholinergic stimulation on the accuracy of item recognition [ $\Delta$ all recognized items (physostigmine) - all recognized items (placebo)].

The multiple regression analysis revealed a significant negative correlation between the number of recognized items at baseline and the effect of cholinergic stimulation on the accuracy of item recognition (Fig. 1a). Thus, subjects with a relatively poor performance at baseline tended to benefit from cholinergic stimulation, whereas subjects who performed better at baseline experienced a negative effect of physostigmine. There was no such effect in spatial context memory retrieval (Fig. 1b).

A repeated measures ANOVA on heart rate with the withinsubject factors measurement time (before drug administration/after scanning) and drug (placebo, physostigmine) neither revealed any main effects of measurement time $\left(F_{(1,17)}=\right.$ $1.103, p=0.308)$ or $\operatorname{drug}\left(F_{(1,17)}=0.401, p=0.534\right)$, nor did it reveal any interaction between drug and measurement time $\left(F_{(1,17)}=0.022, p=0.883\right)$. Side effects in more than one 
a)

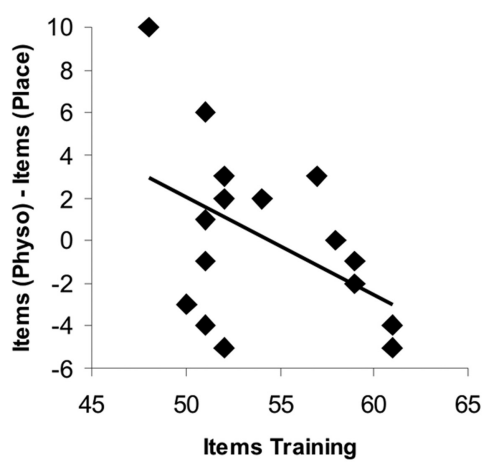

b)

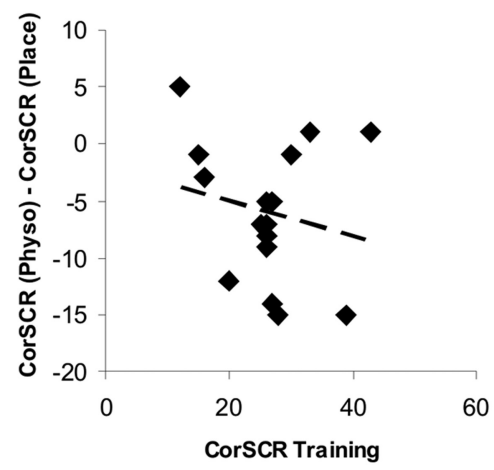

\begin{tabular}{lrrrrrr}
\hline & $\mathbf{d f}$ & $\mathbf{R}^{2}$ & variable & Std. Beta & $\mathbf{t}$ & $\mathbf{p}$ \\
Items(Physo) - Items(Place) & $(2,13)$ & 0.321 & Items (baseline) & -0.745 & -2.469 & $<\mathbf{0 . 0 5}$ \\
& & & Session & -0.430 & -1.427 & 0.177 \\
CorSCR(Physo) - CorSCR(Place) & $(2.13)$ & 0.054 & CorSCR (baseline) & -0.229 & -0.834 & 0.419 \\
& & & Session & -0.098 & -0.359 & 0.725
\end{tabular}

Figure 1. a, Regression analysis of item recognition at baseline [all recognized items (baseline)] and the effect of cholinergic enhancement on item recognition [( $\Delta$ all recognized items (physostigmine) - all recognized items (pla(ebo)]. The trend line depicts a significant correlation. $\boldsymbol{b}$, Regression analysis of spatial context retrieval at baseline [CorSCR (baseline)] and the effect of cholinergic enhancement on spatial context retrieval [ $\Delta$ CorSCR (physostigmine) CorSCR (placebo)]. The dotted trend line depicts a nonsignificant relationship. In both analyses the order of drug administration (session) was introduced as a nuisance covariate.

subject were observed in the physostigmine (plus gylcopyrrholate) session only, including dizziness (11 subjects), nausea (5 subjects), dry mouth (6 subjects), tiredness (3 subjects), and palpitations ( 2 subjects), corresponding to those observed in other studies using the same medication (Bentley et al., 2004, 2008). Several of these side effects (i.e., dry mouth and palpitations) were attributable to glycopyrrholate rather than to physostigmine.

Point-biserial correlation analyses revealed no association between each reported side effect (dizziness, nausea, dry mouth, tiredness, or palpitations) and drug effect on item recognition or on spatial context memory (all $p$ values $>0.05$ ). Furthermore, there was no correlation between baseline performance in item recognition or spatial context memory and subsequent occurrence of side effects (all $p$ values $>0.05$ ).

\section{fMRI data}

Encoding

During encoding, activity in the right superficial nuclei of the amygdala was reduced under cholinergic stimulation compared with placebo, regardless of the success of spatial context encoding (Table 3, Fig. 2a). In the right hippocampal formation, however, there was an interaction effect between drug and spatial context encoding. Post hoc paired $t$ tests revealed that this effect was caused by a significant increase in neural activity during successful versus unsuccessful encoding of spatial context in the physostigmine condition, whereas there was no significant difference in the placebo condition (Table 3, Fig. 2b).

There was no main effect of success of spatial context encoding.

\section{Retrieval}

During retrieval, a main effect of spatial context retrieval was evident in the left amygdala as well as the left and right hip- pocampal formation (Table 3, Fig. 3a). In these regions, successful retrieval was associated with higher activity than unsuccessful retrieval of spatial context.

An interaction effect of cholinergic stimulation with success of spatial context retrieval was evident in the right amygdala (Table 3, Fig. $3 b$ ). Opposite to the effects during encoding, post hoc paired $t$ tests revealed that this interaction was caused by higher neural activity for correctly versus falsely retrieved spatial context during the placebo condition but no such effect in the physostigmine condition.

There was no main effect of cholinergic stimulation during retrieval.

The size of the effects (main or interaction) during encoding or retrieval was not correlated to any of the neuropsychological test data.

\section{Discussion}

We here show differential effects of cholinergic stimulation on neural activity mediating episodic memory encoding or retrieval: an elevation of ACh levels increased neural activity underlying successful encoding of spatial contextual information in the right hippocampal formation but decreased activity for its successful retrieval in the amygdala. These findings confirm the hypothesis that cholinergic transmission specifically promotes encoding of new information at the cost of retrieval at the neural networks level (Hasselmo, 2006). Furthermore, participants who had poorer memory performance at baseline benefited more from cholinergic stimulation than those who had better baseline performance, indicating that a cholinergic deficit contributes to the memory decline even in healthy older subjects.

\section{fMRI data: encoding}

Application of physostigmine increased neural activity, mediating successful encoding of spatial context in the hippocampal formation but decreased neural activity during unsuccessful encoding. Under placebo, there was no such effect, resulting in a significant interaction effect between pharmacological condition and spatial context encoding. These data provide direct evidence for an enhancing effect of cholinergic stimulation on encoding at the neural level in humans and complement previous findings that cholinergic inhibition by administration of the muscarinic receptor antagonist scopolamine reduces neural activity during encoding in the hippocampus and parahippocampal gyrus (Sperling et al., 2002; Schon et al., 2005). It is important to note that the application of physostigmine generally elevated ACh-levels and thus affected both nicotinic and muscarinic receptors. Therefore, an interplay of the activation of both receptor types is likely to contribute to the observed effects: Studies in rodents have shown that activation of presynaptic muscarinic receptors inhibits reciprocal excitatory feedback during encoding in the hippocampal area CA3 (Hasselmo, 1995; Vogt and Regehr, 2001), leading to a reduction of neural interference by ongoing retrieval processes (Hasselmo and Schnell, 1994; Hasselmo 


\begin{tabular}{|c|c|c|c|c|c|c|c|c|}
\hline & Region & Side & $x$ & $y$ & $z$ & Z & $p$ & Effect \\
\hline \multicolumn{9}{|l|}{ Encoding } \\
\hline $\begin{array}{l}\text { Main effect of drug } \\
\text { Main effect of context }{ }^{a}\end{array}$ & Amygdala & $\mathrm{R}$ & 22 & -6 & -9 & 3.56 & $<0.05$ & plac $>$ phys \\
\hline Drug $\times$ context interaction & Hippocampus & $\mathrm{R}$ & 24 & -22 & -19 & 3.72 & $<0.05$ & phys (CorSCE $>$ FalSCE) $>\operatorname{plac}($ CorSCE $>$ FalSCE $)$ \\
\hline 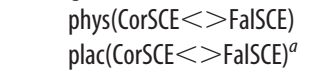 & Hippocampus & $\mathrm{R}$ & 24 & -22 & -19 & 3.57 & $<0.0005$ & phys(CorSCE $>$ FalSCE) \\
\hline \multicolumn{9}{|l|}{ Retrieval } \\
\hline \multirow[t]{4}{*}{ Main effect of context } & Amygdala & L & -20 & -4 & -27 & 4.53 & $<0.0005$ & CorSCR $>$ FalsCR \\
\hline & Hippocampus & L & -12 & -36 & -3 & 3.89 & $<0.05$ & CorsCR $>$ FalsCR \\
\hline & Hippocampus & $\mathrm{R}$ & 24 & -10 & -21 & 3.92 & $<0.05$ & CorSCR $>$ FalSCR \\
\hline & Hippocampus & $\mathrm{R}$ & 30 & -34 & -9 & 3.76 & $<0.05$ & CorSCR $>$ FalSCR \\
\hline $\begin{array}{l}\text { Drug } \times \text { context interaction } \\
\text { phys }(\operatorname{CorSCR}<>\text { FalSCR })^{a}\end{array}$ & Amygdala & $\mathrm{R}$ & 30 & 2 & -29 & 4.36 & $<0.001$ & $\operatorname{plac}(\operatorname{CorSCR}>$ FalSCR $)>$ phys $($ CorSCR $>$ FalSCR $)$ \\
\hline plac(CorSCR $<>$ FalSCR) & Amygdala & $\mathrm{R}$ & 30 & 0 & -29 & 4.59 & $<0.00005$ & plac(CorSCR > FalSCR) \\
\hline
\end{tabular}

List of local maxima for the effects of interest during encoding and retrieval as detected by a repeated-measures ANOVA with the between-subject factor drug and the within-subject factor success of spatial context encoding/retrieval at $p<$ 0.05 FWE-corrected. CorSCE represents activity associated with correct spatial context encoding; FalSCE represents activity associated with false spatial context encoding. CorSCR represents activity associated with correct spatial context retrieval; FaISCR represents activity associated with false spatial context retrieval. Side: left (L) or right (R) hemisphere. Plac, Placebo; phys, physostigmine.

${ }^{a}$ No suprathreshold clusters.

and McGaughy, 2004), whereas activation of nicotinic receptors enhances excitatory input to the hippocampal area CA3 from the entorhinal cortex (Giocomo and Hasselmo, 2005) and from the dentate gyrus (Radcliffe et al., 1999).

The fact that right hippocampal activity predicted subsequent spatial retrieval success only in the physostigmine but not in the placebo condition may be an expression of an incipient cholinergic deficit in the sample of healthy older subjects.

Together, the effects observed in our study are consistent with the concept that increased ACh levels amplify neural selectivity for incoming information (Hasselmo, 2006): the difference between neural activity for successfully and unsuccessfully encoded spatial context was much larger under physostigmine than under placebo conditions. On a neural level, this may have enhanced the efficiency of encoding. At the behavioral level, however, no positive effect of physostigmine on the group mean memory performance was observed. This may be because of a prevailing negative effect of cholinergic stimulation on subsequent retrieval processes.

\section{fMRI data: retrieval}

The present data provide further evidence for a pivotal role of the medial temporal lobe including both the hippocampal formation and the amygdala (presently the left laterobasal nuclei) in retrieval of contextual information. This is in line with previous data implying that the hippocampal formation (Eldridge et al., 2000; Burgess et al., 2001, 2002; Cabeza et al., 2003; Iidaka et al., 2006; Kukolja et al., 2009) and the amygdala (Rutishauser et al., 2008) are not only involved in encoding but also mediate retrieval of context in episodic memory. Previous studies which have investigated amygdalo-hippocampal in-

\section{a) Main effect of drug}
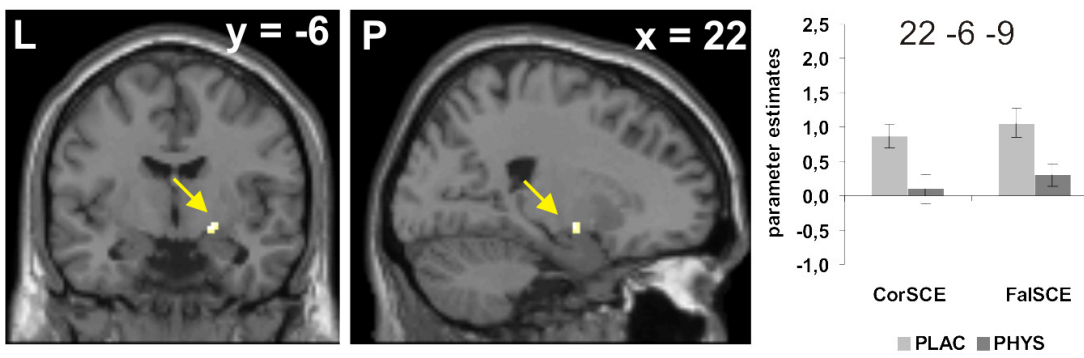

b) Interaction drug $x$ spatial context encoding
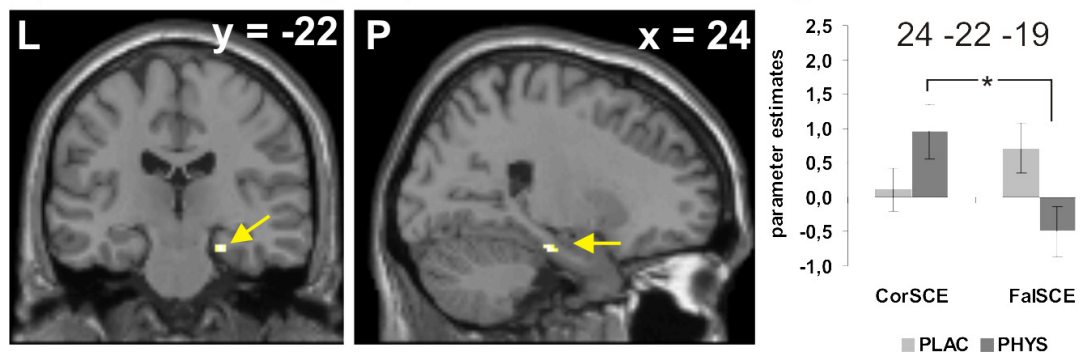

PLAC $\square$ PHYS

Figure 2. Encoding: $\boldsymbol{a}$, Main effect of drug on brain activity. $\boldsymbol{b}$, Interaction effect of drug and spatial context encoding success. Activations are projected on a mean $\mathrm{T} 1$ image provided by SPM 5 . For better visibility, activations are depicted at $p<0.001$, uncorrected. The graphs depict parameter estimates ( $y$-axis) as a function of drug (plac, placebo; phys, physostigmine) and encoding success. CorSCE represents items correctly recognized as previously seen and associated with a correct spatial context judgment during subsequent retrieval; FalSCE represents items correctly recognized as previously seen and associated with a false spatial context judgment during subsequent retrieval. L, left; $P$, posterior.

teractions in memory processes in humans have mostly used long-term and working memory paradigms involving emotional stimuli (Canli et al., 2000; Dolcos et al., 2004; Richardson et al., 2004; LoPresti et al., 2008), suggesting that the amygdala is primarily involved in emotional processing. However, there is strong evidence from human neuroimaging (Dolcos et al., 2004) and single cell recording studies (Rutishauser et al., 2008) that the amygdala is also centrally involved in the encoding and retrieval of nonemotional information.

Elevation of ACh levels during retrieval attenuated activity for successful retrieval of spatial context when compared with placebo. Whereas this effect reached significance only in the right laterobasal amygdaloid nuclei, a nonsignificant tendency 


\section{a) Main effect of spatial context retrieval}
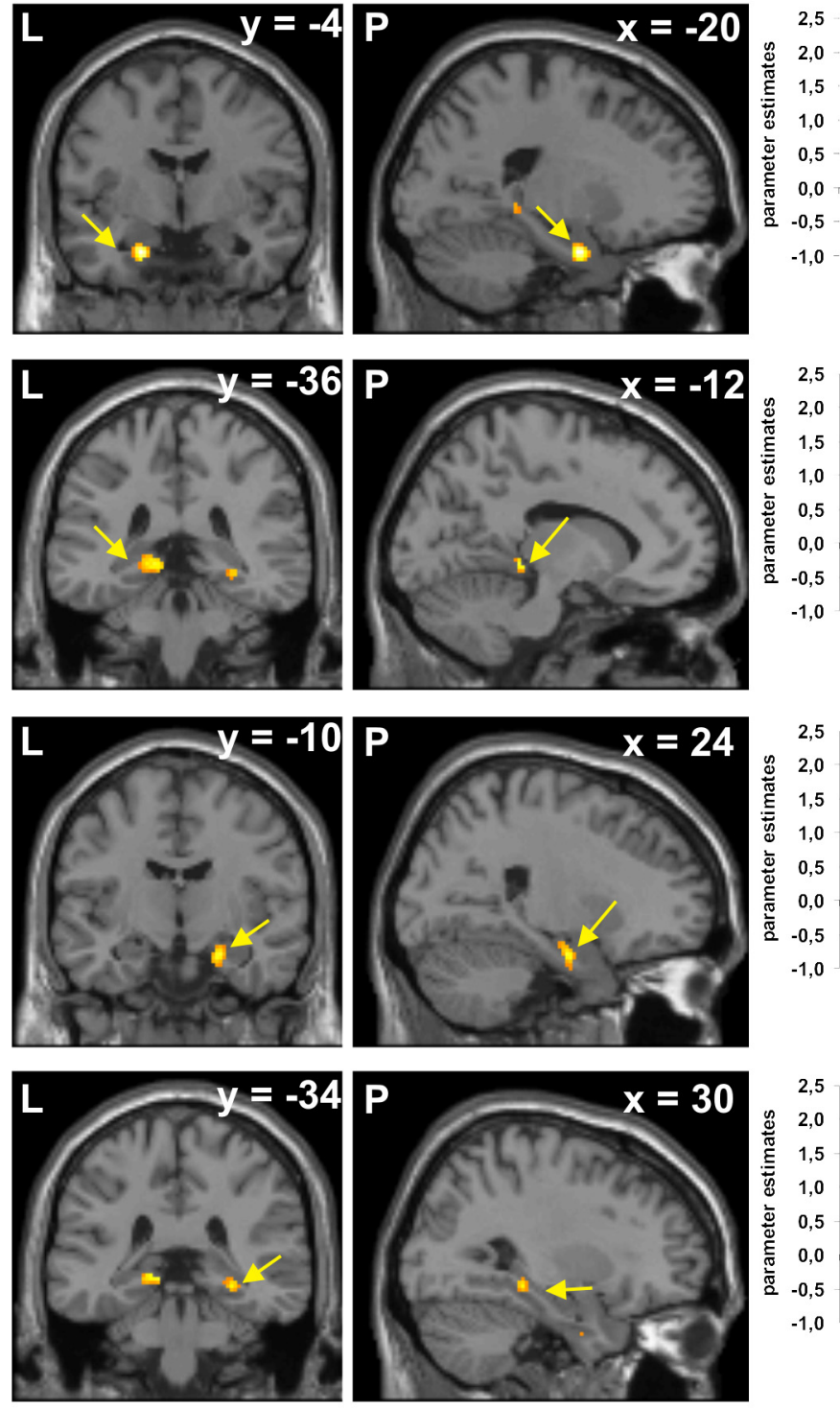

b) Interaction of drug $x$ spatial context retrieval
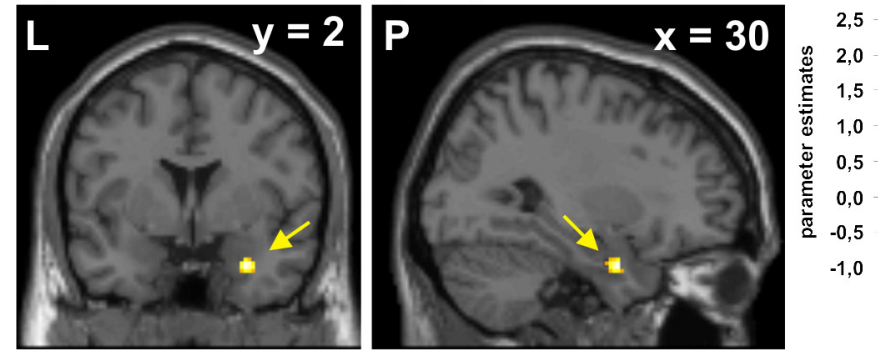

Figure 3. Retrieval: $\boldsymbol{a}$, Main effect of spatial context retrieval success on brain activity. $\boldsymbol{b}$, Interaction effect of drug and spatial context retrieval success. Activations are projected on a mean T1 image provided by SPM 5 . For better visibility, activations are depicted at $p<0.001$, uncorrected. The graphs depict parameter estimates ( $y$-axis) split by drug (plac, placebo; phys, physostigmine) and retrieval success. CorSCR represents items correctly recognized as previously seen and associated with a correct spatial context judgment; FaISCR represents items correctly recognized as previously seen and associated with a false spatial context judgment. L, Left; P, posterior.

for an equivalent effect was also evident in the right cornu ammonis and in the contralateral laterobasal amygdaloid nuclei. This parallels findings that the application of physostigmine during retrieval impaired memory performance in ro-

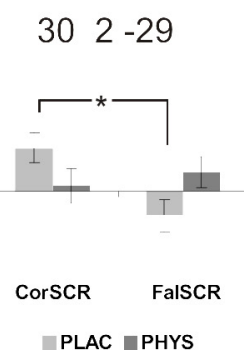

dents (Rogers and Kesner, 2003). The effect might be primarily mediated by ACh binding to muscarinic receptors (Hasselmo and Schnell, 1994), which inhibit neural circuits mediating retrieval (Hasselmo and McGaughy, 2004). Essentially, the observation of a reduction of retrieval-related activity in the medial temporal lobe induced by physostigmine complements the finding of an enhancement of encoding-related processes in our study, as such an effect has been predicted in rodent studies (Hasselmo and McGaughy, 2004). These data strongly support the view that the cholinergic system specifically enhances the processing of incoming information.

\section{fMRI data: general}

pharmacological effects

One important consideration is whether the application of physostigmine might have affected the neurovascular coupling thereby leading to altered fMRI responses when compared with placebo. Speaking against this is the fact that only in the right amygdala but not in other regions such as the contralateral amygdala or the hippocampal formation, a main effect of drug was observed. More importantly, a global effect of physostigmine administration should not have affected differential effects which our conclusions are based on (Thiel, 2003). And finally, the effects of physostigmine administration had opposite effects on differential activation during encoding and retrieval, making a confound resulting from neurovascular effects of physostigmine unlikely.

\section{Behavioral data}

The behavioral results showed that cholinergic stimulation enhanced item memory performance in subjects who had a relatively low baseline performance but impaired such performance in subjects who had high scores at baseline. This suggests that memory deficits within the group of healthy older subjects may be at least in part based on a deficit in the cholinergic system. Whereas an abundance of research studies has concentrated on elucidating neuropharmacological alterations in $\mathrm{AD}$ (Ladner and Lee, 1998; Selkoe, 2002; Cummings et al., 2007; Bentley et al., 2008), there is comparatively little information about the development of neurotransmitter systems in healthy human aging. A cholinergic deficit as the underlying reason for poorer memory performance in healthy older subjects seems plausible, since it is the cholinergic system which primarily degenerates in $\mathrm{AD}$ as the prototypical disease affecting recent episodic memory 
(Bartus et al., 1982; Mesulam, 2004; Cohen et al., 2006; Schliebs and Arendt, 2006), and a decline of cerebral muscarinic receptors has been observed in healthy aging (Norbury et al., 2005). Supporting this view, a recent fMRI study has suggested that the reason for increased persistence and reduced dynamics of hippocampal activity in older subjects is a cholinergic deficit, as mimicked by administration of the muscarinic receptor antagonist scopolamine (Wink et al., 2006).

Further support for a decline of the cholinergic system in normal aging comes from several behavioral studies showing a positive effect of nicotine administration on cognitive functions in elderly subjects (Howe and Price, 2001; Min et al., 2001; White and Levin, 2004). The correlation between baseline performance and the cholinergic effect on memory (ranging from negative to positive results) found in the present study corresponds to a central relationship between several neurotransmitter systems and behavior: the Yerkes-Dodson law states that task performance first improves and then deteriorates with increasing arousal (Yerkes and Dodson, 1908; see Diamond et al., 2007 for review). This relationship has been translated to several neurotransmitter systems, primarily the dopaminergic system (Floresco and Magyar, 2006; Williams and Castner, 2006) but also the noradrenergic (IntroiniCollison and McGaugh, 1986), the cholinergic (Newhouse et al., 2004), and the corticoid system (Lupien and McEwen, 1997; Diamond et al., 2007), indicating that optimal cognitive function requires optimal neurotransmitter levels. According to the Yerkes-Dodson law, aberrations of this optimum either to lower or to higher levels result in decreases of cognitive performance. Translated to our study, it is conceivable that those participants who had low baseline scores also had relatively low cholinergic levels and cholinergic enhancement led to a benefit in memory performance. However, those subjects who did well at baseline bore close to optimal cholinergic levels and further cholinergic enhancement resulted in worse performance.

The finding of a positive correlation between the accuracy in (visual) item recognition with immediate recall in the (auditory) VLMT corroborates the assumption that performance in the present task was dependent on the general integrity of the memory functions in each individual.

Although nonsignificant, the deleterious effects of physostigmine on spatial context memory observed in our study might be primarily caused by reduced retrieval efficiency. Data from rodent and human studies suggest that high ACh levels enhance encoding by presynaptic muscarinic inhibition of excitatory feedback circuits and thereby facilitate feedforward neuronal input (Hasselmo, 2006; Hasselmo and Giocomo, 2006). This mechanism may, however, disrupt the circuits mediating retrieval and consolidation (Gais and Born, 2004). It is important to note that the observed behavioral effects are probably attributable to specific cholinergic actions in the CNS rather than to peripheral or central side effects as no correlation with side effects was found.

The fact that no significant effect of drug on the behavioral performance during encoding was observed is probably caused by the relatively low difficulty level of the task (item discrimination) as proven by the high accuracy and may thus be explained by a ceiling effect.

\section{Conclusions}

In conclusion, the present study provides evidence for a differential effect of cholinergic stimulation on encoding and retrieval of items and their spatial context. Whereas neural activity during encoding of spatial context was enhanced, neural activity mediating retrieval was attenuated. These findings strengthen the suggestion, mainly derived from studies in rodents, that cholinergic stimulation facilitates the processing of incoming information but interferes with neural circuitry mediating retrieval. Furthermore, the present results, showing that participants with lower memory performance at baseline tend to benefit from cholinergic stimulation whereas those with higher performance do not, indicate that memory deficits in healthy older subjects are the result of a cholinergic deficit.

\section{References}

Amunts K, Kedo O, Kindler M, Pieperhoff P, Mohlberg H, Shah NJ, Habel U, Schneider F, Zilles K (2005) Cytoarchitectonic mapping of the human amygdala, hippocampal region and entorhinal cortex: intersubject variability and probability maps. Anat Embryol 210:343-352.

Atri A, Sherman S, Norman KA, Kirchhoff BA, Nicolas MM, Greicius MD, Cramer SC, Breiter HC, Hasselmo ME, Stern CE (2004) Blockade of central cholinergic receptors impairs new learning and increases proactive interference in a word paired-associate memory task. Behav Neurosci 118:223-236.

Bartus RT, Dean RL 3rd, Beer B, Lippa AS (1982) The cholinergic hypothesis of geriatric memory dysfunction. Science 217:408-414.

Beck AT, Hautzinger M, Bailer M, Worall H, Keller F (1995) Beck-DepressionsInventar (BDI) 2, überarbeitete Auflage. Göttingen, Germany: Hogrefe.

Bentley P, Husain M, Dolan RJ (2004) Effects of cholinergic enhancement on visual stimulation, spatial attention, and spatial working memory. Neuron 41:969-982.

Bentley P, Driver J, Dolan RJ (2008) Cholinesterase inhibition modulates visual and attentional brain responses in Alzheimer's disease and health. Brain 131:409-424.

Benton AL, Hamsher K (1976) Multilingual aphasia examination. Iowa City, IA: University of Iowa.

Buccafusco JJ, Letchworth SR, Bencherif M, Lippiello PM (2005) Longlasting cognitive improvement with nicotinic receptor agonists: mechanisms of pharmacokinetic-pharmacodynamic discordance. Trends Pharmacol Sci 26:352-360.

Burgess N, Maguire EA, Spiers HJ, O’Keefe J (2001) A temporoparietal and prefrontal network for retrieving the spatial context of lifelike events. Neuroimage 14:439-453.

Burgess N, Maguire EA, O'Keefe J (2002) The human hippocampus and spatial and episodic memory. Neuron 35:625-641.

Cabeza R, Locantore JK, Anderson ND (2003) Lateralization of prefrontal activity during episodic memory retrieval: evidence for the productionmonitoring hypothesis. J Cogn Neurosci 15:249-259.

Canli T, Zhao Z, Brewer J, Gabrieli JD, Cahill L (2000) Event-related activation in the human amygdala associates with later memory for individual emotional experience. J Neurosci 20:RC99.

Cansino S, Maquet P, Dolan RJ, Rugg MD (2002) Brain activity underlying encoding and retrieval of source memory. Cereb Cortex 12:1048-1056.

Cohen RM, Carson RE, Filbey F, Szczepanik J, Sunderland T (2006) Age and APOE-epsilon4 genotype influence the effect of physostigmine infusion on the in-vivo distribution volume of the muscarinic-2-receptor dependent tracer [18F]FP-TZTP. Synapse 60:86-92.

Crook TH 3rd, Feher EP, Larrabee GJ (1992) Assessment of memory complaint in age-associated memory impairment: the MAC-Q. Int Psychogeriatr 4:165-176.

Cummings JL, Doody R, Clark C (2007) Disease-modifying therapies for Alzheimer disease: challenges to early intervention. Neurology 69:1622-1634.

Diamond DM, Campbell AM, Park CR, Halonen J, Zoladz PR (2007) The temporal dynamics model of emotional memory processing: a synthesis on the neurobiological basis of stress-induced amnesia, flashbulb and traumatic memories, and the Yerkes-Dodson law. Neural Plast 60803:1-33.

Dolcos F, LaBar KS, Cabeza R (2004) Interaction between the amygdala and the medial temporal lobe memory system predicts better memory for emotional events. Neuron 42:855-863.

Eickhoff SB, Stephan KE, Mohlberg H, Grefkes C, Fink GR, Amunts K, Zilles K (2005) A new SPM toolbox for combining probabilistic cytoarchitectonic maps and functional imaging data. Neuroimage 25:1325-1335.

Eldridge LL, Knowlton BJ, Furmanski CS, Bookheimer SY, Engel SA (2000) 
Remembering episodes: a selective role for the hippocampus during retrieval. Nat Neurosci 3:1149-1152.

Floresco SB, Magyar O (2006) Mesocortical dopamine modulation of executive functions: beyond working memory. Psychopharmacology 188:567-585.

Freo U, Ricciardi E, Pietrini P, Schapiro MB, Rapoport SI, Furey ML (2005) Pharmacological modulation of prefrontal cortical activity during a working memory task in young and older humans: a PET study with physostigmine. Am J Psychiatry 162:2061-2070.

Friston KJ, Holmes A, Worsley KJ, Poline JB, Frith CD, Frackowiak RSJ (1995) Spatial registration and normalisation of images. Hum Brain Mapp 3:165-189.

Furey ML, Pietrini P, Haxby JV (2000) Cholinergic enhancement and increased selectivity of perceptual processing during working memory. Science 290:2315-2319.

Gais S, Born J (2004) Low acetylcholine during slow-wave sleep is critical for declarative memory consolidation. Proc Natl Acad Sci U S A 101:2140-2144.

Giocomo LM, Hasselmo ME (2005) Nicotinic modulation of glutamatergic synaptic transmission in region CA3 of the hippocampus. Eur J Neurosci 22:1349-1356.

Hasselmo ME (1995) Neuromodulation and cortical function: modeling the physiological basis of behavior. Behav Brain Res 67:1-27.

Hasselmo ME (2006) The role of acetylcholine in learning and memory. Curr Opin Neurobiol 16:710-715.

Hasselmo ME, Giocomo LM (2006) Cholinergic modulation of cortical function. J Mol Neurosci 30:133-135.

Hasselmo ME, McGaughy J (2004) High acetylcholine levels set circuit dynamics for attention and encoding and low acetylcholine levels set dynamics for consolidation. Prog Brain Res 145:207-231.

Hasselmo ME, Schnell E (1994) Laminar selectivity of the cholinergic suppression of synaptic transmission in rat hippocampal region CA1: computational modeling and brain slice physiology. J Neurosci 14:3898-3914.

Howe MN, Price IR (2001) Effects of transdermal nicotine on learning, memory, verbal fluency, concentration, and general health in a healthy sample at risk for dementia. Int Psychogeriatr 13:465-475.

Iidaka T, Matsumoto A, Nogawa J, Yamamoto Y, Sadato N (2006) Frontoparietal network involved in successful retrieval from episodic memory. Spatial and temporal analyses using fMRI and ERP. Cereb Cortex 16:1349-1360.

Introini-Collison IB, McGaugh JL (1986) Epinephrine modulates longterm retention of an aversively motivated discrimination. Behav Neural Biol 45:358-365.

Kessler J, Markowitsch HJ, Denzler PE (1990) Mini-Mental-Status-Test (MMST). Dt. Fassung. Göttingen, Germany: Hogrefe.

Kukolja J, Thiel CM, Wolf OT, Fink GR (2008) Increased cortisol levels in cognitively challenging situations are beneficial in young but not older subjects. Psychopharmacology 201:293-304.

Kukolja J, Thiel CM, Wilms M, Mirzazade S, Fink GR (2009) Ageing-related changes of neural activity associated with spatial contextual memory. Neurobiol Aging 30:630-645.

Ladner CJ, Lee JM (1998) Pharmacological drug treatment of Alzheimer disease: the cholinergic hypothesis revisited. J Neuropathol Exp Neurol 57:719-731.

Lanctôt KL, Herrmann N, Yau KK, Khan LR, Liu BA, LouLou MM, Einarson TR (2003) Efficacy and safety of cholinesterase inhibitors in Alzheimer's disease: a meta-analysis. CMAJ 169:557-564.

Lehrl S (1995) Mehrfachwahl-Wortschatz-Test (MWT-B). Erlangen: Straube.

Levin ED, McClernon FJ, Rezvani AH (2006) Nicotinic effects on cognitive function: behavioral characterization, pharmacological specification, and anatomic localization. Psychopharmacology 184:523-539.

Light LL (1991) Memory and aging: four hypotheses in search of data. Annu Rev Psychol 42:333-376.

LoPresti ML, Schon K, Tricarico MD, Swisher JD, Celone KA, Stern CE (2008) Working memory for social cues recruits orbitofrontal cortex and amygdala: a functional magnetic resonance imaging study of delayed matching to sample for emotional expressions. J Neurosci 28:3718-3728.

Lupien SJ, McEwen BS (1997) The acute effects of corticosteroids on cognition: integration of animal and human model studies. Brain Res Brain Res Rev 24:1-27.

Mahalanobis PC (1936) On the generalised distance in statistics. Proc Natl Inst Sci India 2:49-55.
McGaugh JL (2004) The amygdala modulates the consolidation of memories of emotionally arousing experiences. Annu Rev Neurosci 27:1-28.

McGaugh JL, McIntyre CK, Power AE (2002) Amygdala modulation of memory consolidation: interaction with other brain systems. Neurobiol Learn Mem 78:539-552.

McIntyre CK, Power AE, Roozendaal B, McGaugh JL (2003) Role of the basolateral amygdala in memory consolidation. Ann NY Acad Sci 985:273-293.

Mesulam M (2004) The cholinergic lesion of Alzheimer's disease: pivotal factor or side show? Learn Mem 11:43-49.

Mesulam MM (1996) The systems-level organization of cholinergic innervation in the human cerebral cortex and its alterations in Alzheimer's disease. Prog Brain Res 109:285-297.

Mesulam MM, Geula C (1988) Nucleus basalis (Ch4) and cortical cholinergic innervation in the human brain: observations based on the distribution of acetylcholinesterase and choline acetyltransferase. J Comp Neurol 275:216-240

Mesulam MM, Mufson EJ, Levey AI, Wainer BH (1983) Cholinergic innervation of cortex by the basal forebrain: cytochemistry and cortical connections of the septal area, diagonal band nuclei, nucleus basalis (substantia innominata), and hypothalamus in the rhesus monkey. J Comp Neurol 214:170-197.

Min SK, Moon IW, Ko RW, Shin HS (2001) Effects of transdermal nicotine on attention and memory in healthy elderly non-smokers. Psychopharmacology 159:83-88.

Mufson EJ, Ginsberg SD, Ikonomovic MD, DeKosky ST (2003) Human cholinergic basal forebrain: chemoanatomy and neurologic dysfunction. J Chem Neuroanat 26:233-242.

Naveh-Benjamin M (2000) Adult age differences in memory performance: tests of an associative deficit hypothesis. J Exp Psychol Learn Mem Cogn 26:1170-1187.

Newhouse PA, Potter A, Singh A (2004) Effects of nicotinic stimulation on cognitive performance. Curr Opin Pharmacol 4:36-46.

Norbury R, Travis MJ, Erlandsson K, Waddington W, Owens J, Pimlott S, Ell PJ, Murphy DG (2005) In vivo imaging of muscarinic receptors in the aging female brain with (R,R)[123I]-I-QNB and single photon emission tomography. Exp Gerontol 40:137-145.

Oldfield RC (1971) The assessment and analysis of handedness: the Edinburgh inventory. Neuropsychologia 9:97-113.

Radcliffe KA, Fisher JL, Gray R, Dani JA (1999) Nicotinic modulation of glutamate and GABA synaptic transmission of hippocampal neurons. Ann N Y Acad Sci 868:591-610.

Rasch BH, Born J, Gais S (2006) Combined blockade of cholinergic receptors shifts the brain from stimulus encoding to memory consolidation. J Cogn Neurosci 18:793-802.

Rawlings JO, Pantula SG, Dickey DA (2001) Applied regression analysis: a research tool. Heidelberg: Springer.

Richardson MP, Strange BA, Dolan RJ (2004) Encoding of emotional memories depends on amygdala and hippocampus and their interactions. Nat Neurosci 7:278-285.

Rogers JL, Kesner RP (2003) Cholinergic modulation of the hippocampus during encoding and retrieval. Neurobiol Learn Mem 80:332-342.

Rutishauser U, Schuman EM, Mamelak AN (2008) Activity of human hippocampal and amygdala neurons during retrieval of declarative memories. Proc Natl Acad Sci U S A 105:329-334.

Satzger W, Hampel H, Padberg F, Bürger K, Nolde T, Ingrassia G, Engel RR (2001) [Practical application of the CERAD test battery as a neuropsychological dementia screening test]. Nervenarzt 72:196-203.

Schacter DL, Wagner AD (1999) Medial temporal lobe activations in fMRI and PET studies of episodic encoding and retrieval. Hippocampus 9:7-24

Schliebs R, Arendt T (2006) The significance of the cholinergic system in the brain during aging and in Alzheimer's disease. J Neural Transm 113:1625-1644.

Schon K, Atri A, Hasselmo ME, Tricarico MD, LoPresti ML, Stern CE (2005) Scopolamine reduces persistent activity related to long-term encoding in the parahippocampal gyrus during delayed matching in humans. J Neurosci 25:9112-9123.

Selkoe DJ (2002) Alzheimer's disease is a synaptic failure. Science 298:789-791.

Spencer WD, Raz N (1995) Differential effects of aging on memory for content and context: a meta-analysis. Psychol Aging 10:527-539.

Sperling R, Greve D, Dale A, Killiany R, Holmes J, Rosas HD, Cocchiarella A, 
Firth P, Rosen B, Lake S, Lange N, Routledge C, Albert M (2002) Functional MRI detection of pharmacologically induced memory impairment. Proc Natl Acad Sci U S A 99:455-460.

Talairach J, Tournoux P (1988) Co-planar stereotaxic atlas of the human brain. 3-Dimensional proportional system: an approach to cerebral imaging. Stuttgart, Germany: Thieme.

Tewes U (1991) Hamburg-Wechsler-Intelligenztest für Erwachsene, Revision (HAWIE-R). Bern, Switzerland: Huber.

Thiel CM (2003) Cholinergic modulation of learning and memory in the human brain as detected with functional neuroimaging. Neurobiol Learn Mem 80:234-244.

Thiel CM, Henson RN, Dolan RJ (2002) Scopolamine but not lorazepam modulates face repetition priming: a psychopharmacological fMRI study. Neuropsychopharmacology 27:282-292.

Trinh NH, Hoblyn J, Mohanty S, Yaffe K (2003) Efficacy of cholinesterase inhibitors in the treatment of neuropsychiatric symptoms and functional impairment in Alzheimer disease: a meta-analysis. JAMA 289:210-216.

Vogt KE, Regehr WG (2001) Cholinergic modulation of excitatory synaptic transmission in the CA3 area of the hippocampus. J Neurosci 21:75-83.

White HK, Levin ED (2004) Chronic transdermal nicotine patch treatment effects on cognitive performance in age-associated memory impairment. Psychopharmacology 171:465-471.

Williams GV, Castner SA (2006) Under the curve: critical issues for elucidating D1 receptor function in working memory. Neuroscience 139:263-276.

Wink AM, Bernard F, Salvador R, Bullmore E, Suckling J (2006) Age and cholinergic effects on hemodynamics and functional coherence of human hippocampus. Neurobiol Aging 27:1395-1404.

Worsley KJ, Friston KJ (1995) Analysis of fMRI time-series revisited-again. Neuroimage 2:173-181.

Yerkes RM, Dodson JD (1908) The relation of strength of stimulus to rapidity of habit-formation. J Comp Neurol Psychol 18:459-482. 